\title{
Increasing environmental compatibility of metal production
}

\author{
Rashid Sharapov ${ }^{1, *}$, Evgeny Kharlamov ${ }^{1}$, and Valentina Yadykina $^{2}$ \\ ${ }^{1}$ Moscow State University of Civil Engineering, Yaroslavskoe shosse, 26, Moscow, 129337, Russia \\ ${ }^{2}$ Belgorod state technological university named after V. G. Shukhov, Kostyukova str., 46, Belgorod, \\ 308012, Russia
}

\begin{abstract}
Building materials production generates a large amount of harmful substances poisoning the atmosphere. One of the major sources polluting cities environment is metallurgical industry. Concentration is one of the most important processes where empty components are extracted from the rock. During ore concentration, an increasing number of manmade wastes are generated; they pollute the air and huge area around the factories discharging these wastes. This reduces both space for people to live and place for cities to function and develop. It should be noted that metal production enterprises have accumulated billions of tons of industrial wastes (tailings) that include a large amount of iron-containing materials and rocks; these can be used as building materials, for example, when preparing fine-grained concrete as a mineral powder as well as in construction of roads, houses, in paint production, etc.
\end{abstract}

\section{Introduction}

Currently, mining and processing plants mainly use wet separation patterns and drum separators [1, 2]. PBM type drum separators (Fig. 1) with multipolar system made of permanent magnets are used for wet magnetic separation of highly magnetic ores and are made in three versions: with direct-flow, counterflow and semi-counterflow baths $[3,4]$. At the direct-flow separators the entire feed volume passes through the extraction area, while at the semi-counterflow and counterflow separators non-magnetic part passes through it. Direct flow separators are used for materials with particle size of $6 \mathrm{~mm}$ or less, counterflow separators - for materials with particle size of 2(3) $\mathrm{mm}$ or less, semi-counterflow separators - for materials with particle size of $0.3 \mathrm{~mm}$ or less $[5,6]$.

Separator 26-SB (Fig. 2) is a counterflow magnetic drum unit designed to concentrate drains of ball mills and classifiers [7]. Separator 26-SB operating principle is as follows: feed goes through the pipe to the separator charging box, then it is carried by two feed branches to the feed tray and under the rotating drum. When exposed to magnetic field, magnetic particles are attracted to the drum and moved to the magnetic system edge, where they are separated from the drum and discharged. Non-magnetic particles together with bulk of water are discharged through the tail pipe.

* Corresponding author: ptdm_zavkaf@ mail.ru 

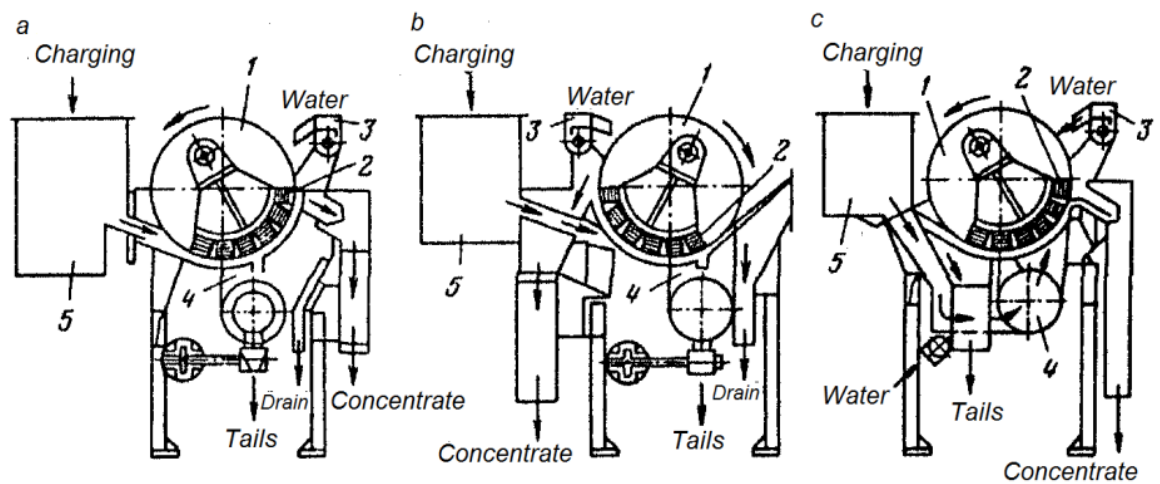

Fig. 1. Drum separator PBM-90/250 for wet ore concentration: $a$ - with direct-flow bath; $b$ - with counterflow bath; $c$ - with a semi-counterflow bath.

The above magnetic separators are for wet separation which have the following disadvantages: presence of aqueous medium with higher resistance as compared to the air; aqueous medium strongly counteracts flow of magnetic and nonmagnetic particles, consequently, some magnetic particles are lost with nonmagnetic ones; when magnetic materials are separated, there is no magnetic flocculation (formation of reinforced aggregates due to mutual attraction of magnetized particles); need for feeding medium water (wastewater).

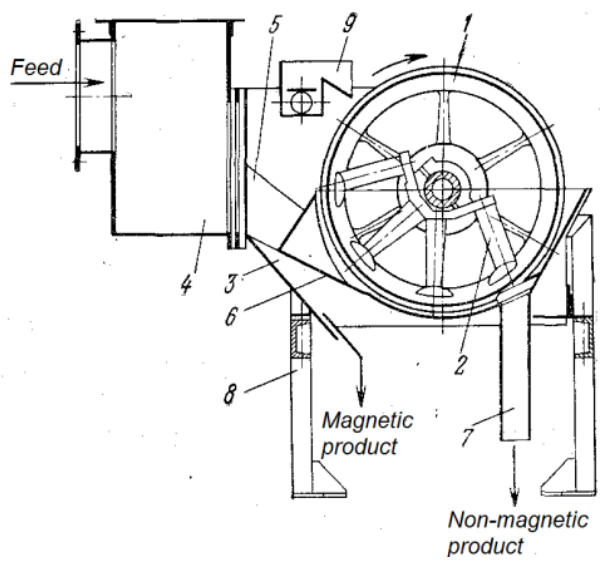

Fig. 2. Counterflow magnetic drum separator 26-SB: 1 - drum; 2 - magnetic system; 3 - bath; 4 charging box; 5 - feed branches; 6 - feed tray; 7 - tail branch; 8 - frame; 9 - splatter.

Waste and circulating water are sourced from drains of dehydrating, desliming, flushing devices and concentration tailings. Polluting impurities in them are solid particles, hardness salts, heavy metal ions and organic substances [8, 9]. Raw waste water containing suspended impurities and aggregates causes environmental system disturbance with all negative consequences: rivers become shallow and dry, vegetation withers, surrounding life fades $[10,11]$. To treat waste water and harmful impurities, mechanical, chemical, physicochemical and biochemical methods are used. All this causes additional costs and rise in finished products price.

Dry separation method is alternative to wet separation one. From an environmental point of view, dry magnetic separation is the most acceptable method. When no feeding medium is used, you get significant water saving, there is no need for waste water treatment or for sludge dumps, which occupy considerable areas and adversely affect the atmosphere 
and lithosphere $[12,13]$. Dry separation is the least energy intensive method as well, since it is based on the natural property of iron ores magnetic attraction.

\section{Methods}

Efficiency of concentration tailings dry separation at metallurgical production enterprise may be increased through combination of metal particles magnetic extraction and separated material air turning processes $[9,14]$.

An experimental facility for exploration of two-component mixture separation process was designed considering these requirements. An operational facility photo is shown in Fig. 3.

The experimental facility consists of the following main elements: belt conveyor 1 , magnetic particle discharge conveyor 2 , scraper conveyor 3 , transport chute 4 , charging device 5, stabilization system 6 , control panel 7, magnetic particle discharge area 8 and non-magnetic particle discharge area 9 . The belt conveyor is designed to remove magnetic particles from mixture. A block of magnets is hinge-mounted inside the belt conveyor traction loop along the return belt. The belt conveyor is installed at an angle against the transport tray. This belt conveyor position creates an alternating magnetic field intensity against the mixture moving in the transport tray, from the minimum intensity at the beginning of extraction to the maximum one at the end drum. Particle extraction process takes place, accordingly, during the entire period while mixture is moving under the belt conveyor active section, at the beginning of which highly magnetic particles and then particles with decreasing magnetization are extracted.

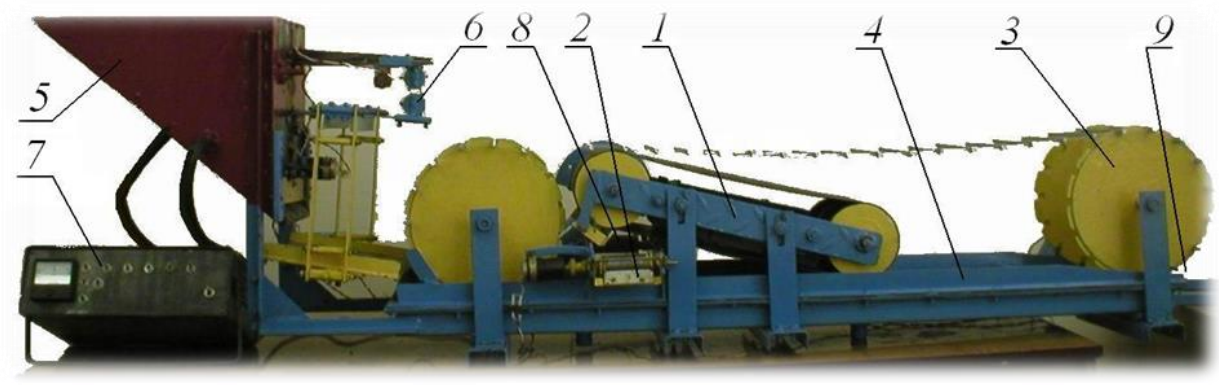

Fig. 3. Experimental facility: 1 - belt conveyor; 2 - magnetic particles discharge conveyor; 3 scraper conveyor; 4 - tray; 5 - charging device; 6 - stabilization system; 7 - control panel; 8 magnetic particles discharge area; 9 - non-magnetic particles discharge area.

The scraper conveyor is designed to stabilize mixture movement along the transport tray until non-magnetic particles discharging, and it does not interfere with separation of magnetic particles from the mixture. The scraper conveyor contains a two-chain traction assembly made of non-magnetic materials with scrapers fixed to it. The transport tray is made with the bottom in the form of a box. The transport tray upper plane is made of an airpermeable porous material. The air passing through the porous material forms a compressed air layer between the porous material surface and mixture, as a result, the air passes through the mixture and creates fluidization effect. Mixture separation into fractions in fluidization condition and increase in particles mobility increases their possible capture by magnetic field, contributes increasing in magnetic particles extraction coefficient, reduces possible particles accumulation on the partition and coefficient of their resistance to scrapers movement.

Fluidized bed dry separator functions as follows. Working medium (air) is fed through the branches to the transport tray hollow elements at the fluidized bed dry separator and charging device, then it passes through the porous material and creates compressed air layer 
in the contact area of the material with the porous material; this compressed air layer reduces cohesion coefficient by 3-6 times and ensures material steady movement. The initial mixture of magnetic and non-magnetic particles is charged into the charging device hopper. The mixture then passes through the slots not closed by slide valve in the charging device hopper front wall to the vibrating tray. The vibrating tray reciprocates perpendicularly to mixture flow. Initial mixture is evenly distributed on it over charging width. The distributed initial mixture is fed to the fluidized bed dry separator transport tray and is moved by conveyor scrapers to the non-magnetic particles area. The return belt moves in the mixture counterflow and attracts magnetic particles from the mixture, with particle extraction process as follows. First, highly magnetic particles, then, the particles by their decreasing magnetization are extracted. The extracted particles held by magnetic field move with the belt to the point where they are loaded onto the magnetic particle discharge conveyor, which conveys them outside the facility. Non-magnetic particles are conveyed further to the non-magnetic particles discharge area.

\section{Results}

Fluidized bed dry separator extraction capacity depends on magnetic system characteristics, physical and mechanical properties of the particles in the separated mixture, and also on working area geometric parameters $[15,16]$. Magnetic particles extraction coefficient $\eta$ is determined by the ratio of their mass flows at the entrance to the active area $G\left(x_{A}\right)$ and at the output from it $G\left(x_{B}\right)$ (Fig. 4):

$$
\eta=1-\frac{G\left(x_{B}\right)}{G\left(x_{A}\right)}
$$

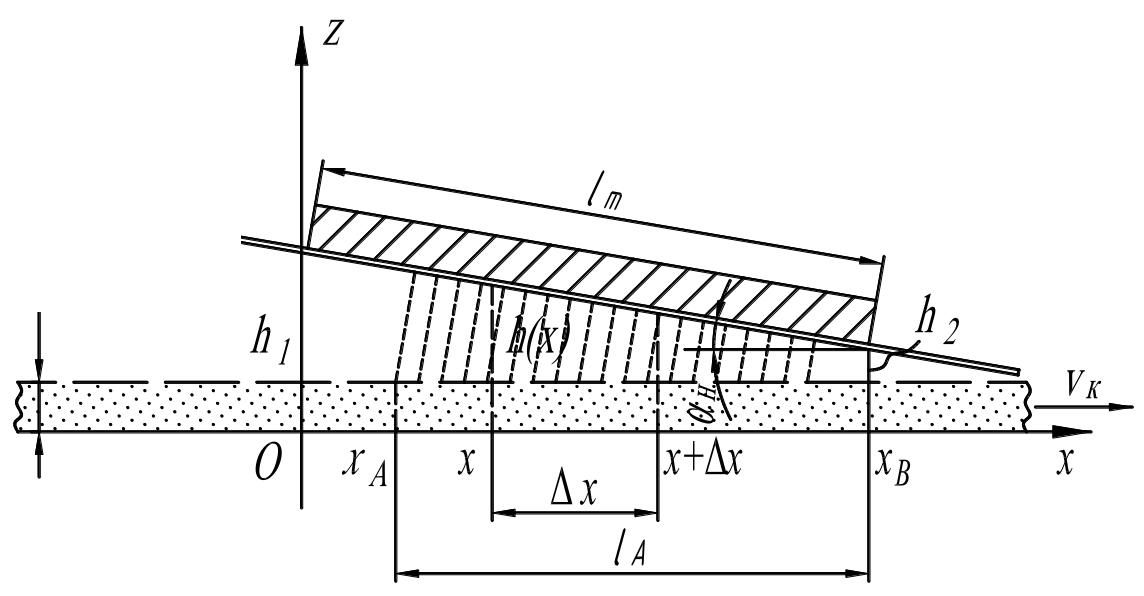

Fig. 4. Computational scheme to derive magnetite particles extraction coefficient.

When particles are extracted in the separation area, an aerodisperse flow of magnetic particles occurs and moves along the fluidized bed dry separator working area with the velocity of scraper conveyor $v_{k}$. Magnetic particles concentration in this flow is distributed along the working area height very unevenly: it sharply decreases from the maximum value in the fluidized bed to the minimum value in the deposition area on the transport conveyor belt.

Let's consider a part of the fluidized bed dry separator working area between the cross sections passing through the points $x$ and $x+\Delta x$ (see Fig. 4). Magnetic particles deposition 
results in their concentration decrease from $C(x)$ to $C(x+\Delta x)$. Magnetic particles material balance equation for the working area selected part:

$$
G(x)-G(x+\Delta x)=\chi C(x) v_{\mathrm{oc}} b \frac{\Delta x}{\cos \alpha}=\chi \frac{G(x) v_{\mathrm{oc}} \Delta x}{h(x) v_{k} \cos \alpha_{\text {н }}}
$$

Here $\Delta x / \cos \alpha$ is a conveyor belt segment corresponding to $\Delta x, G(x)$ is the magnetic particles mass flow passing through the working area cross section corresponding to the coordinate $x$ :

$$
G(x)=C(x) b h(x) v
$$

where $h(x)$ is the height of this section:

$$
h(x)=h_{1}-x \operatorname{tg} \alpha_{\mathrm{H}}
$$

where $v_{1}$ is the precipitation velocity of particles on the transport conveyer belt; where $b$ is the cross section width; $\alpha$ is the angle of scraper conveyor; $v$ is the separeted material speed.

By expressing the change in magnetic particles mass flow through its differential, we obtain:

$$
G(x)-G(x+\Delta x)=-(G(x+\Delta x)-G(x)) \approx-d G(x)
$$

and by dividing the variables in the equation (1) we obtain:

$$
\frac{d G}{G}=-\frac{\chi v_{1} d x}{v\left(h_{1}-x \operatorname{tg} \alpha\right) \cos \alpha}
$$

By integrating the equation (6) we obtain:

$$
\frac{d G}{G}=-\frac{\chi v_{1} d x}{v\left(h_{1}-x \operatorname{tg} \alpha\right) \cos \alpha}
$$

The ratio (7) results in:

$$
\frac{G\left(x_{B}\right)}{G\left(x_{A}\right)}=\left(\frac{h_{1}-x_{B} \operatorname{tg} \alpha}{h_{1}-x_{A} \operatorname{tg} \alpha}\right)^{\frac{x v_{1}}{v \sin \alpha}}
$$

By substituting the equation (8) to the formula (1) we obtain the ratio for magnetic particles fractional extraction coefficient:

$$
\eta(d)=1-\left(\frac{h_{2}}{h_{2}+l_{A} \operatorname{tg} \alpha}\right)^{\frac{\chi v_{1}}{v \sin \alpha}}
$$

For the working area $l_{A}$ active section length included in the formula (6) we obtain:

$$
l_{A}=-\frac{h_{2}-1,115 \delta}{\operatorname{tg} \alpha}-\frac{1}{2 c \sin \alpha} l_{n}\left(\frac{\rho g \cos \alpha}{\mu_{0} \chi c H_{0}^{2}}\right) .
$$

For the experimental fluidized bed dry separator $\left(h_{2}=0.015 \mathrm{~m}, \delta=0.003-0.013 \mathrm{~m}, v=\right.$ $0.012-0.028 \mathrm{mps}, \alpha_{\mathrm{H}}=10-20^{\circ}, c=26.17 \mathrm{~m}^{-1}, H_{0}=37 \mathrm{kApm}, \chi_{\mathrm{h}}=6.25, \rho_{\mathrm{M}}=5,260 \mathrm{kgpm}^{3}$, $\mathrm{F}_{\mathrm{M}}=1.65, g=9.81 \mathrm{mps}^{2}, \mu_{0}=4 \pi \cdot 10^{-7}(\mathrm{~kg} \cdot \mathrm{m}) /\left(\mathrm{s}^{2} \cdot \mathrm{A}^{2}\right), d_{i}=\{2,7 ; 8,3 ; 17,2 ; 31,7 ; 55\} \mu \mathrm{m}, \mu_{\mathrm{d}}=$ 
$\left.1.8 \cdot 10^{-5} \mathrm{~Pa} \cdot \mathrm{s}, \delta_{1}=0.003 \mathrm{~m}\right)$ parameter $l_{A}$ and velocity of particles deposition onto the transport conveyor belt $v_{1}$ (formula (10)) take on the following form:

$$
\begin{gathered}
l_{A}=-\frac{0,015-1,115 \delta}{\operatorname{tg} \alpha}-\frac{\ln (0,183 \cos \alpha)}{52,34 \sin \alpha}, \\
v_{1}=4,472 \cdot 10^{-4} d^{2},
\end{gathered}
$$

where particle size $d$ should be given in $\mu \mathrm{m}$.

Taking into account the formulas (11) and (12), the equation (9) can be rewritten as follows:

$$
\eta(d)=1-\left(\frac{0,015}{1,115 \delta-\frac{\ln (0,183 \cos \alpha)}{52,34 \cos \alpha}}\right)^{\frac{4,472 \cdot 10^{-4} d^{2} x}{v \sin \alpha}}
$$

Total magnetite particles extraction coefficient $\eta$ is determined by fractional coefficients $\eta\left(\bar{d}_{i}\right)$ and particles dispersed composition:

$$
\eta=\sum_{i} \eta\left(\bar{d}_{i}\right) f_{i}
$$

Comparison of the total magnetite particles extraction coefficient theoretical and experimental values shows that the value of distribution inhomogeneity coefficient for their concentration in the fluidized bed dry separator working area $\chi$ depends mainly on the thickness of separated mixture in the initial, bound state $\delta$. Processing of experimental data resulted in the following dependence:

$$
\chi(\sigma)=50 \delta^{2}-0.15 \delta+0.0224
$$

By substituting the formula (15) to the (13) we obtain a final equation for the fractional magnetic particles extraction coefficient in the experimental fluidized bed dry separator:

$$
\eta(d)=1-\left(\frac{0,015}{1,115 \delta-\frac{\ln (0,183 \cos \alpha)}{52,34 \cos \alpha}}\right)^{\frac{4,472 \cdot 10^{-4} d^{2}\left(50 \delta^{2}-0,15 \delta+0,0224\right)}{v \sin \alpha}}
$$

It results from the formulas (6) and (7) that the magnetic particles extraction coefficient depends on their physical and mechanical properties (density, size, shape, permeability), magnetic system characteristics (its sizes, magnetic field intensity, pole pitch), constructive and technological parameters of fluidized bed dry separator (sizes, magnetic system angle of inclination, separated mixture layer thickness, scraper conveyor velocity), as well as on air medium properties (temperature, viscosity).

To confirm the above theory, we conducted an experimental study. The material used is the Lebedinskiy mining and processing plant tailings. During the experiment, the following parameters were changed: thickness of separating material layer $\delta$; separating material movement velocity $v$; belt conveyor angle of inclination $\alpha$ and magnetic field intensity $\mathrm{H}$. 


\section{Conclusions}

Fig. 5 shows dependence of influence of separated material layer thickness $\delta$ on separation efficiency $\eta$ at different values of separated material movement velocity $v$, constant belt conveyor angle of inclination $\alpha=15^{\circ}$ and magnetic field intensity $H=40 \mathrm{kApm}$. Fig. 5 demonstrates that all presented dependences are of increasing nature, i.e. as separated material layer thickness, determined by the parameter $\delta$, increases, so does separation efficiency. When analyzing the graphs shown in Fig. 5, we can conclude that the separation efficiency maximum value $\eta$ in the experiment is achieved at an iron-containing composition movement velocity of $v=0.02 \mathrm{mps}$ and layer thickness $\delta$ equal to $14 \mathrm{~mm}$, and is $\eta=91.23 \%$.

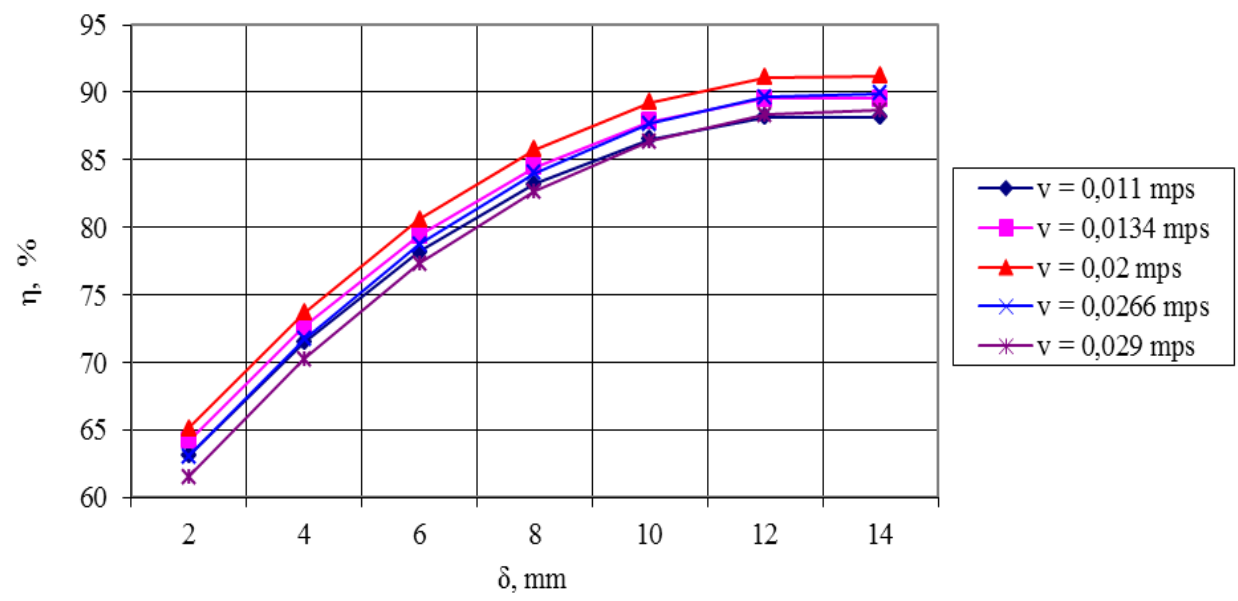

Fig. 5. Dependence of separation efficiency $\eta$ on the separated material layer thickness $\delta$ at different values of separated material movement velocity $v$, conveyor belt constant angle of inclination $\alpha=15^{\circ}$, and magnetic field intensity $H=40 \mathrm{kApm}$.

Fig. 6 shows dependence of influence of separated material movement velocity $v$ on separation efficiency $\eta$ at different values of belt conveyor angle of inclination $\alpha$, separated material layer constant thickness $\delta=8 \mathrm{~mm}$, and magnetic field intensity kApm. Fig. 6 demonstrats that all the dependencies presented are of extreme nature in the studied range of factors variation.

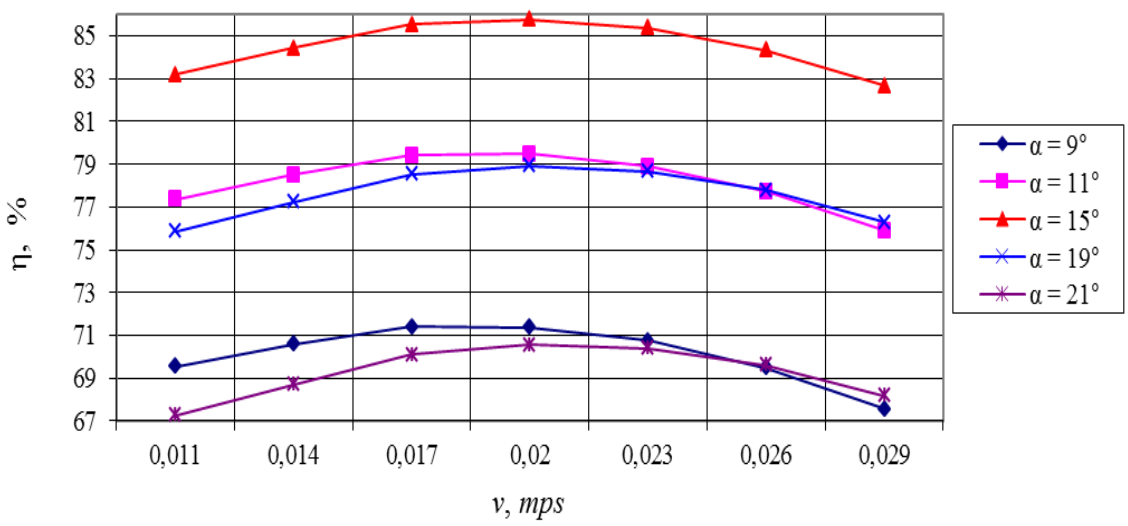

Fig. 6. Dependence of separation efficiency $\eta$ on separated material movement velocity $v$ at different 
values of belt conveyor angle of inclination $\alpha$, constant layer thickness $\delta=8 \mathrm{~mm}$, and magnetic field intensity $\mathrm{H}=40 \mathrm{kApm}$.

When analyzing the graphs shown in Fig. 6, we can conclude that the maximum separation efficiency value $\eta$ is reached at separated material movement velocity of $v=0.02 \mathrm{mps}$, belt conveyor angle of inclination of $\alpha=15^{\circ}$ and is $\eta=85.77 \%$.

To increase metallurgical production environmental compatibility, facilities operated employing particle extraction magnetic and separated material air turning principle can be used to separate concentration tailings with a high degree of efficiency.

An experimental fluidized bed separator unit was designed and constructed for experiment.

The experimental studies confirm that the fluidized bed dry separator has a higher efficiency as compared to conventional separators and exceeds $90 \%$.

\section{References}

1. R.P. Bhagat, P.N. Pathak, International Journal of Mineral Processing 47, 213-217 (1996)

2. T. Kaljuvee, R. Kuusik, M. Veiderma, International Journal of Mineral Processing 43, 113-121 (1995)

3. M. Lovás, I. Murová, A. Mockovciaková, N. Rowson, S. Jakabský, Separation and Purification Technology 31, 291-299 (2003)

4. H. Zhang, L. Chen, J. Zeng, L. Ding, J. Liu, Separation Science and Technology 50, 1689-1694 (2015)

5. V.A. Simakov, V.E. Isaev, Inorganic Materials 51, 1438-1441 (2015)

6. A.M. Turkenich, E.S. Lapshin, V.I. Dudnik, Magnetic and Electrical Separation 8, 8187 (1997)

7. V.E. Potapenko, D.I. Suvorova, V.V. Tyuryukhanova, Refractories and Industrial Ceramics 22, 146-148 (1981)

8. V.F. Skorokhodov, S.P. Mesyats, S.P. Ostapenko, Eurasian Mining, 1, 58-60 (2011)

9. E.V. Kharlamov, R.R. Sharapov, V.V. Yadykina, International Journal of Applied Engineering Research 10, 45108-45114 (2015)

10. I.V. Melik-Gaykazov, Yu.G Druz., V.A. Shchetkin, Yu.A. Komyagina, V.F. Skorokhodov, Eurasian Mining 2, 58-60 (2012)

11. E. Korol, N. Shushunova, Procedia Engineering, World Multidisciplinary Civil Engineering-Architecture-Urban Planning Symposium, WMCAUS 2016, 1820-1826 (2016)

12. J. Oshitani, G.V. Franks, M. Griffin, Advanced Powder Technology 21, 573-577 (2010)

13. K.E. Anatolevna, K.P. Borisovich, International Journal of Applied Engineering Research 10, 43456-43460 (2015)

14. R. Sharapov, P. Kapyrin, S. Lozovaya, V. Yadykina, A. Agarkov, MATEC Web of Conferences, 5th International Scientific Conference "Integration, Partnership and Innovation in Construction Science and Education", IPICSE 2016 (2016)

15. N.S. Sevryugina, MATEC Web of Conferences 117, 00151 (2017)

16. N.S. Sevryugina, E.A. Volkov, E.P. Litovchenko, Science 8, 179-185 (2014) 